\title{
Persistência do Canal Arterial: o Papel das Próteses Oclusoras
} Ver artigo relacionado
na página 15
Valmir F. Fontes ${ }^{1}$, Carlos A. C. Pedra ${ }^{1}$
A persistência do canal arterial é uma anomalia que incide em 7 a 10\% das cardiopatias congênitas como lesão isolada. O tratamento cirúrgico vem sendo praticado desde 1938, quando Gross e Hubbard $^{1}$ descreveram o primeiro caso operado com sucesso. Nos dias de hoje, o procedimento cirúrgico é simples e seguro, com baixíssima taxa de morbimortalidade. A abordagem é feita através de uma toracotomia látero-posterior esquerda, onde o canal é seccionado e seus cotos suturados. Outra alternativa de uso rotineiro é a ligadura do canal com ligaclip através de uma minitoracotomia. Contudo, trata-se de uma cirurgia, com passagem pela UTI e tempo de hospitalização de dois a quatro dias.

O fechamento por dispositivos oclusores passou a ser uma alternativa mais simples e oferece resultados equivalentes aos do tratamento cirúrgico. O procedimento pode ser ambulatorial ou necessitar internação hospitalar por 24 horas, sem incisão cirúrgica, minimizando a ansiedade do paciente e de seus familiares.

O fechamento percutâneo do canal arterial foi introduzido inicialmente por Porstmann et al. ${ }^{2}$, que criaram um dispositivo liberado por via transfemoral, o plug de Ivalon, cujo desenho obedecia à configuração anatômica do canal. O método mostrou limitações quanto ao peso e à idade do paciente, factível apenas para maiores de 5 anos. Rashkind et al.$^{3-5}$ desenvolveram uma prótese, inicialmente de disco único e minúsculos ganchos de fixação, que posteriormente passou a ser composta por dois discos sem ganchos, utilizando-se para o seu implante um cateter especial de liberação controlada, tendo como via de acesso a veia femoral. A prótese apresentava-se em dois tamanhos, 12 e $17 \mathrm{~mm}$, capaz de ocluir canais de 2 a $8 \mathrm{~mm}$ de diâmetro e foi universalmente utilizada, inclusive no Brasil ${ }^{6}$. Sua retirada do mercado deveu-se ao desenvolvimento de outros dispositivos mais eficientes. Grifka et al. ${ }^{7}$ e o grupo de Sideris ${ }^{8}$ introduziram suas próteses na década de 90, as quais não ganharam aceitação entre os intervencionistas. Cambier et al. ${ }^{9}$ passaram a utilizar molas helicoidais de Gianturco, técnica que se tornou mundialmente aceita e reservada para canais arteriais até $3 \mathrm{~mm}$ de diâmetro, com constrição em seu trajeto, presente nos tipos A, D e E da classificação de Krichenko et al. ${ }^{10}$. A via de acesso é, usualmente, a artéria femoral e a prótese é de liberação livre. Existem espirais de liberação controlada, entre elas as do tipo Flipper e, mais recentemente, o Nit-Occlud, capaz de ocluir canais de 2 a $5 \mathrm{~mm}$ de diâmetro. Os resultados são excelentes e reproduzidos em todos os Laboratórios de Hemodinâmica do mundo ${ }^{11,12}$.

Nos dias de hoje, a prótese mais efetiva é a Amplatzer, especialmente desenhada para oclusão de canal arterial persistente. É uma prótese auto-expansível, com formato de um cogumelo ou bala, construída com uma malha fina de nitinol e fibras de poliéster no seu interior, que facilitam a trombose. Possui um disco de retenção 4 a $6 \mathrm{~mm}$ maior que o dispositivo que fica alojado na ampola aórtica do canal arterial. A prótese é utilizada em canais com diâmetro maior que $2 \mathrm{~mm}$ e com tamanhos de 5-4 a $16-14 \mathrm{~mm}$. A anatomia mais favorável é aquela que exibe uma constrição no seu trajeto $(\mathrm{A}, \mathrm{D} \text { e } \mathrm{E})^{10}$; o tipo $\mathrm{C}$ pode ser ocluído e o tipo B, em janela, é o mais problemático. A ampola aórtica do canal no tipo B é muito rasa e a prótese pode provocar algum grau de obstrução aórtica. Para tal situação, recomenda-se uma prótese de duplo disco como a Amplatzer, utilizada no fechamento de CIA ou CIV.

A prótese Amplatzer foi introduzida no Brasil, em 1998, no Instituto Dante Pazzanese de Cardiologia, é um dispositivo de simples manejo e de aprendizado fácil. No nosso meio, o seu uso é limitado devido ao alto custo. O dispositivo é hoje usado universalmente e os resultados são excelentes a curto, médio e longo prazos, com índice de oclusão próximos aos 100\%.

Em estudo multicêntrico realizado nos Estados Unidos $^{13}$, envolvendo 484 pacientes portadores de canal arterial persistente, 439 foram selecionados para o implante e obteve-se sucesso em 435. A análise de 360 casos por ecocardiografia, um ano após a oclusão,

\footnotetext{
1 Instituto Dante Pazzanese de Cardiologia, São Paulo, SP.

Correspondência: Dr. Valmir F. Fontes. Av. Dr. Dante Pazzanese, 500 - 14ㅇandar - Ibirapuera - São Paulo - SP - CEP 04012-180

Tel: (11) 5085-6114 ou 5085-6214 • e-mail: valmirfontes@dantepazzanese.org.br

Recebido em: 08/02/2007 • Aceito em: 14/02/2007
} 
mostrou que apenas um doente apresentava discreto fluxo artério-venoso residual, ou seja, a oclusão completa ocorreu em 99,7\% destes casos.

Masura et al. ${ }^{14}$, entre os pioneiros a empregar a prótese, mostraram resultados excelentes, mas advertem para dificuldades técnicas em crianças de baixo peso e canais calibrosos. No Brasil, a experiência inicial de duas instituições foi publicada por Simões et al. ${ }^{15}$, que verificaram ser uma técnica segura e efetiva, comparável aos resultados da literatura internacional.

As complicações do implante são raras, ocorrendo usualmente durante o procedimento ou nas primeiras 24 horas pós-implante. A literatura registra um único óbito relacionado à embolização precoce do dispositivo para a aorta descendente ${ }^{16}$. A embolização em médio e longo-prazos não está registrada na literatura. Os fluxos artério-venosos residuais de alta velocidade podem provocar hemólise mecânica, mas esta é uma complicação rara. A formação de trombo ou tromboembolismo também não é relatada na literatura, mas muitos autores recomendam o uso de aspirina $(5 \mathrm{mg} / \mathrm{kg}$ ) por 6 meses após o implante, em todos os pacientes.

A endocardite infecciosa não tem sido descrita, porém existe potencial para tal, especialmente, nos casos com fluxo artério-venoso residual, justificando a profilaxia com antibióticos.

A obstrução da aorta secundária à protusão do disco aórtico tem sido observada em poucos casos; é raramente vista na artéria pulmonar esquerda. A detecção é feita por meio da ecocardiografia com Doppler, valorizando-se o aumento da velocidade do fluxo. A obstrução tem sido descrita em canais muito angulados em que a prótese não se acomoda adequadamente na ampola aórtica do canal. Este inconveniente foi resolvido pela indústria (AGA), que desenhou um dispositivo angulado (angled ADO) para esta variedade anatômica. Outra possibilidade de obstrução ocorre no canal tipo janela (B).

No momento atual, considerando as próteses existentes no mercado brasileiro, achamos que as molas helicoidais de Gianturco são uma boa opção para canais até 2,8-3,0mm, especialmente dos tipos A, D e E de Krichenko, sendo efetiva e de baixo custo. As próteses helicoidais de liberação controlada são uma outra opção, especialmente a Nit-Occlud, indicada para canais de 2 a $5 \mathrm{~mm}$ de diâmetro, porém de custo elevado. Finalmente, a prótese Amplatzer, seguramente a mais confortável para se trabalhar, permite ocluir canais maiores que $2 \mathrm{~mm}$ de diâmetro, mas também tem como inconveniente o seu alto custo.

\section{REFERÊNCIAS BIBLIOGRÁFICAS}

1. Gross RE, Hubbard JP. Surgical ligation of a patent ductus arteriosus. Report of first successful case. JAMA 1939;112:729-31.

2. Porstmann $\mathbf{W}$, Wierny $L$, Warnke $H$. The closure of the patent ductus arteriosus without thoractomy (preliminary report). Thoraxchir Vask Chir 1967;15:199-203.

3. Rashkind WJ, Cuaso CC. Transcatheter closure of a patent ductus arteriosus: successful use in a $3.5 \mathrm{~kg}$ infant. Pediatr Cardiol 1979;1:63-5

4. Rashkind WJ. Transcatheter treatment of congenital heart disease. Circulation 1983;67:711-6.

5. Rashkind WJ, Mullins CE, Hellenbrand WE, Tait MA. Nonsurgical closure of patent ductus arteriosus: clinical application of the Rashkind PDA Occluder System. Circulation 1987;75: 583-92.

6. Fontes VF, Pedra CA, Esteves CA, Braga SL, Pedra SR, Santana MV, et al. Cierre percutáneo del conducto arterioso com la técnica de Rashkind. Experiência inicial del Instituto Dante Pazzanese de Cardiologia - São Paulo, Brasil. Arch Inst Cardiol Méx 1996;66:129-37.

7. Grifka RG, Vincent JA, Nihill MR, Ing FF, Mullins CE. Transcatheter patent ductus arteriosus closure in an infant using the Gianturco-Grifka Vascular Occlusion Device. Am J Cardiol 1996;78:721-3.

8. Rao PS, Sideris EB, Haddad J, Rey C, Hausdorf G, Wilson $A D$, et al. Transcatheter occlusion of patent ductus arteriosus with adjustable buttoned device. Initial clinical experience. Circulation 1993;88:1119-26.

9. Cambier PA, Kirby WC, Wortham DC, Moore JW. Percutaneous closure of the small (less than $2.5 \mathrm{~mm}$ ) patent ductus arteriosus using coil embolization. Am J Cardiol 1992;69:815-6.

10. Krichenko A, Benson LN, Burrows P, Moes CA, McLaughlin P, Freedom RM. Angiographic classification of the isolated, persistently patent ductus arteriosus and implications for percutaneous catheter occlusion. Am J Cardiol 1989;63:877-80.

11. Magee AG, Huggon IC, Seed PT, Qureshi SA, Tynan M. Association for European Cardiology.Transcatheter coil occlusion of the arterial duct; results of the European Registry. Eur Heart J 2001;22:1817-21.

12. Wang JK, Liau CS, Huang JJ, Hsu KL, Lo PH, Hung JS, et al. Transcatheter closure of patent ductus arteriosus using Gianturco coils in adolescents and adults. Catheter Cardiovasc Interv 2002;55:513-8.

13. Pass RH, Hijazi Z, Hsu DT, Lewis V, Hellenbrand WE. Multicenter USA Amplatzer patent ductus arteriosus occlusion device trial: initial and one-year results. J Am Coll Cardiol 2004;44:513-9.

14. Masura J, Tittel P, Gavora P, Podnar T. Long-term outcome of transcatheter patent ductus arteriosus closure using Amplatzer duct occluders. Am Heart J 2006;151:755.e7-755.e10.

15. Simões LC, Pedra CA, Esteves CA, Camargo R, Braga SL, Loureiro $\mathrm{P}$, et al. Fechamento percutâneo do canal arterial com prótese Amplatzer. Experiência no Brasil. Arq Bras Cardiol $2001 ; 77: 520-31$.

16. Faella HJ, Hijazi ZM. Closure of the patent ductus arteriosus with the Amplatzer PDA device: immediate results of the international clinical trial. Catheter Cardiovasc Interv $2000 ; 51: 50-4$ 